\title{
Engajamento no trabalho, saúde mental e personalidade em oficiais de justiça
}

\author{
Natália dos Santos Dalanhol \\ Universidade Federal de Ciências da Saúde de Porto Alegre, RS, Brasil \\ Clarissa Pinto Pizarro de Freitas \\ Universidade Salgado de Oliveira, RJ, Brasil \\ Wagner de Lara Machado \\ Pontifícia Universidade Católica de Campinas, SP, Brasil \\ Claudio Simon Hutz \\ Universidade Federal do Rio Grande do Sul, RS, Brasil \\ Ana Claudia Souza Vazquez \\ Universidade Federal de Ciências da Saúde de Porto Alegre RS, Brasil
}

\begin{abstract}
Resumo
O engajamento no trabalho é definido como uma experiência positiva associada ao bem-estar e desempenho profissional. O objetivo deste estudo foi investigar suas relações com saúde mental e personalidade em servidores do judiciário. A amostra foi composta por 82 oficiais de justiça ( $57,3 \%$ do sexo feminino) com idade média 47,4 anos ( $D P=9,8$ anos), que responderam um questionário sócio-demográfico e instrumentos validados dos construtos estudados. Foram realizadas análises descritivas, correlações, regressão hierárquica (método stepwise) e análise de rede. Os resultados mostraram que os oficiais de justiça da amostra apresentam problemas de saúde mental e que a energia vital é o principal preditor do engajamento. Ademais, o engajamento associa-se negativamente com distúrbios psiquiátricos menores e de forma indireta com neuroticismo. Estes achados apontam para a importância de promover a saúde mental e o engajamento destes servidores através de intervenções em nível organizacional, pois estes fatores são influenciados pelo ambiente e organização laboral.
\end{abstract}

Palavras-chave: saúde do trabalhador; fatores psicossociais; personalidade; bem-estar; psicologia positiva.

\section{Work engagement, mental health and personality in justice officers}

\begin{abstract}
Work engagement is defined as a positive experience related to well-being and job performance. The aim of this study was to investigate its associations with mental health and personality in judiciary workers. The sample consisted of 82 justice officers ( $57.3 \%$ female) with mean age of 47.4 years $(S D=9.8$ years), who answered a socio-demographic survey and validated instruments of the constructs studied. Descriptive analysis, correlations analysis, hierarchical regression (stepwise method) and network analysis were performed. The results showed that the justice officers have mental health problems and that vital energy was the main predictor of engagement. Also, engagement associates negatively with minor psychiatric disorders and, indirectly, with neuroticism. These findings indicate the importance of promoting mental health and engagement of these workers through interventions at the organizational level, because these factors are influenced by environment and labor organization.
\end{abstract}

Keywords: worker's health; psychosocial factors; personality; well-being; positive psychology.

\section{Compromiso con el trabajo, salud mental y personalidad en oficiales de justícia}

\section{Resumen}

El compromiso en el trabajo es definido como una experiencia positiva asociada al bien-estar y desarrollo profesional. El objetivo de este estudio fue investigar sus relaciones con la salud mental y la personalidad de los funcionarios del sector judicial. El muestreo fue compuesto por 82 oficiales de justicia ( $57,3 \%$ mujeres) con edad media de 47,4 años ( $\mathrm{SD}=9,8$ años), que han respondido a un cuestionario socio-demográfico e instrumentos validados de los constructos estudiados. Fueron realizados análisis descriptivos, análisis de correlaciones, regresión jerárquica (metodo stepwise) y analisis de red. Los resultados mostraron que los oficiales de justicia presentan problemas de salud mental siendo la energía vital el principal predictivo del compromiso. Además, el compromiso se correlaciona negativamente con los disturbios psiquiátricos menores y con neuroticismo de manera indirecta. Estas conclusiones apuntan para la importancia en promover la salud mental y el compromiso de estos trabajadores a través de intervenciones en nivel organizacional, ya que estos factores están influenciados por el ambiente y la organización del trabajo.

Palabras clave: salud del trabajador; factores psicosociales; personalidad; bien-estar; psicología positiva. 


\section{Introdução}

As transformações ocorridas nos ambientes econômicos e sociais atuais possibilitaram que o trabalho se constitua como fonte de prazer e satisfação (Schaufeli, Dijkstra, \& Vazquez, 2013). Esta visão mais positiva implica em um movimento que se concentra na compreensão e na promoção do funcionamento saudável; ou seja, nos aspectos positivos da saúde do trabalhador. Neste contexto, estudos têm demonstrado que o engajamento no trabalho pode atuar como um promotor de maiores índices de bemestar e desempenho organizacional (Schaufeli, 2013; Schaufeli et al., 2013). O engajamento é um estado mental positivo caracterizado por um alto nível de energia que desencadeia sensação de bem-estar, preenchimento e identificação com o trabalho; sendo caracterizado por três dimensões específicas: vigor, dedicação e concentração (Schaufeli, 2013; Schaufeli et al., 2013).

O engajamento é analisado pelo modelo teórico de Recursos e Demandas do Trabalho (Modelo RDT) (Schaufeli \& Taris, 2014). Neste, o bem-estar, a saúde e a motivação do trabalhador são resultados do balanceamento ótimo entre recursos e demandas das características do trabalho. Altas demandas de trabalho desgastam recursos físicos e mentais do trabalhador, e podem causar esgotamento de energia e problemas de saúde, como a Síndrome de Burnout. Enquanto recursos de trabalho apresentam potencial motivacional para conduzir as pessoas ao alto desempenho em suas atividades por meio do engajamento. Dado o risco de experienciar resultados negativos ao lidar com demandas e condições adversas de trabalho, os recursos de trabalho se caracterizam como fatores de proteção que mantêm as pessoas saudáveis (Schaufeli \& Taris, 2014). Desta forma, o engajamento apresenta relações positivas com a saúde e o bem-estar do trabalhador (Schaufeli, 2013).

No Modelo RDT, características individuais foram incluídas como uma terceira variável, além dos recursos e demandas do trabalho, que são identificadas como recursos pessoais do trabalhador (Schaufeli \& Taris, 2014). Os recursos pessoais referem-se às habilidades do indivíduo de controlar e impactar seu ambiente de forma bem sucedida. Dentre os mais citados na literatura estão o otimismo, a autoeficácia, a autoestima e os traços de personalidade (e.g., extroversão, conscienciosidade, neuroticismo, amabilidade) (Schaufeli \& Taris, 2014). Referente aos traços de personalidade foi observado que o engajamento apresentou: a) correlação negativa com neuroticismo (Lin et al., 2016; Sulea, Van Beek, Sarbescua, Virga, \& Schaufeli, 2015; Zis,
Anagnostopoulos, \& Artemiadis, 2016); (b) correlação positiva com extroversão (Maas \& Spinath, 2012) e conscienciosidade (Maas \& Spinath, 2012; Lin et al., 2016; Sulea et al., 2015); (c) já com a amabilidade foram observadas relações positivas (Sulea et al., 2015) e negativas (Maas \& Spinath, 2012).

Segundo o Ministério da Previdência Social (2015), a saúde mental está entre as principais causas atribuídas ao afastamento do trabalho no Brasil, sendo grande a prevalência dos transtornos de humor. Os indicadores fornecidos em 2014 pelo Tribunal de Justiça do Rio Grande do Sul referentes às licenças de saúde para servidores e magistrados nos anos de 2009 a 2011 demonstram uma média de 45.722 dias de licença por ano, o que equivaleria à média de 5,71 dias ao ano por trabalhador. O índice do CID-10 de letra F, "transtornos mentais e comportamentais", é a razão com maior ocorrência. A categoria dos oficiais de Justiça aparece como a principal acometida por agravos à saúde que requerem licenças do trabalho, sendo a subclassificação F31 (transtorno afetivo bipolar) a mais comum, seguida por F33 (transtorno depressivo recorrente) (Ministério da Previdência Social, 2015). Tais dados reforçam a necessidade de estudos a respeito das condições de trabalho servidores do Judiciário em geral, e dos oficiais de Justiça, em particular, de modo a melhor compreender os vínculos entre a especificidade do seu trabalho e as relações com o processo saúde/doença.

Estudos realizados com profissionais do Judiciário e das áreas relacionadas à segurança têm demonstrado como estes profissionais estão expostos a condições de trabalho precárias (Merlo, Dornelles, Bottega, \& Trentini, 2012; Pereira \& Assunção, 2007; Tavares, 2003). O trabalho dos oficiais de Justiça é realizar a intermediação entre os juízes e os cidadãos, comunicando as ordens (mandados) a serem acatadas. Realizam trabalho de campo, sendo comum a necessidade de se dirigir mais de uma vez a um mesmo endereço, o que acaba trazendo mais pressão, devido à sobrecarga no volume de mandados com prazos estipulados de cumprimento. As situações frequentemente geram uma carga emocional, especialmente em mandados como o de despejo, busca e apreensão de menores, separação de corpos e de prisão. Os cidadãos em situação de constrangimento podem reagir agredindo o oficial física ou verbalmente. Portanto, os trabalhadores tornam-se constantemente apreensivos devido aos riscos presentes (Pereira \& Assunção, 2007).

O estudo de Tavares (2003) evidencia a representação social do trabalho em servidores do Tribunal Judiciário e demonstra que o sofrimento destes se expressa pelo medo, sentimento de autodesvalorização, 
desesperança, desalento, contaminação do pensamento e do sono por conteúdos do trabalho e adoecimentos somatopsicológicos. O autor identificou ainda problemas referentes à organização do trabalho e às políticas organizacionais que repercutem negativamente na saúde dos funcionários.

Um estudo realizado com 78 oficiais de Justiça Federal de Porto Alegre demonstrou resultados que apontam para a falta de reconhecimento do trabalho pela instituição como principal problema, além do desrespeito em relação ao exercício da atividade e o trabalho solitário (Merlo et al., 2012). O reconhecimento pode ser produzido por colegas, contudo deveria ocorrer também pela chefia imediata, neste caso o juiz. No entanto, em razão da atividade exercida pelos oficiais ser geralmente solitária, raramente ocorrem contatos diretos com juízes. Não havendo reconhecimento, inviabiliza-se que a instituição compreenda as necessidades inerentes à função. Ademais, elevada prevalência de distúrbios psiquiátricos menores (50,7\%) foi observada nesta população (Merlo et al., 2012). Demais dificuldades relacionadas ao exercício desta profissão como abandono, estresse ocupacional, sobrecarga emocional e funcional, inabilidade de gestão e falta de motivação para o trabalho foram relatadas por servidores do judiciário em estudo realizado na mesma região (Pizzinato, Carlotto, Cé \& Conceição, 2014).

Tendo em vista as particularidades e riscos psicossociais presentes no trabalho do Judiciário, o presente estudo objetivou analisar as relações entre as características pessoais, a saúde mental e índices de engajamento dos oficiais de justiça. Desta forma, esta pesquisa pretende investigar as relações entre o engajamento no trabalho, o traço de personalidade neuroticismo e a saúde mental de oficiais de justiça em Porto Alegre e região metropolitana.

\section{Método}

\section{Participantes}

A população do estudo é composta por 200 oficiais de justiça do Tribunal de Justiça S (TJRS), de Porto Alegre e região metropolitana. A amostra, não probabilística, foi composta por 82 sujeitos ( $41 \%$ da população) que atuam nas instâncias de 10 e 2o Grau.

As análises descritivas evidenciaram que a maioria dos participantes $(57,3 \% ; n=47)$ pertencia ao sexo feminino, com idade média de 47,4 anos $(D P=9,8$, amplitude de 21 a 68 anos). Em relação ao estado civil, 18,3\% $(n=15)$ eram solteiros, $53,7 \%(n=44)$ casados, $13,4 \%(n=11)$ separados ou divorciados, $1,2 \%$ $(n=1)$ viúvos, $12,2 \%(n=10)$ estavam em uma união estável e 1,2\% $(n=1)$ não responderam. Dentre estes profissionais, 4,9\% $(n=4)$ possuíam o ensino médio completo, 66 com ensino superior, sendo 57,3\% $(n=47)$ com curso de nível superior completo e $23,2 \%(n=19)$ em andamento ou incompleto. Foi observado que $13,4 \%(n=11)$ dos profissionais possuíam ao menos um curso de Pós-Graduação e 1,2\% $(n=1)$ preferiram não responder. Grande parte atua em Porto Alegre, sendo $32,9 \%$ no Tribunal de Justiça $(n=27)$ e $46,3 \%$ nas $1^{\text {a }}$ e $2^{a}$ comarcas $(n=38)$. Além destes, $15,9 \%(n=13)$ participantes são da 3a comarca da cidade de Canoas, $1,2 \%$ pertencem a $4^{\text {a }}$ comarca, $2,4 \%(n=2)$ da $10^{\text {a }}$ comarca e $1,2 \%(n=1)$ não responderam. Quanto ao vínculo laboral, 69 profissionais $(84,1 \%)$ informaram serem servidores estatutários de 10 Grau, 4,9\% $(n=4)$ informaram serem servidores estatutários de $2^{\circ}$ Grau, $3,7 \%(n=3)$ funcionários de CLT de 1 o Grau, $2,4 \%$ $(n=2)$ magistrados de 1 o Grau.

\section{Instrumentos}

Escala Utrecht de Engajamento no Trabalho (Vazquez, Magnan, Pacico, Hutz \& Schaufeli, 2015): composta por 17 itens, englobando os três fatores do engajamento (Vitalidade, Dedicação e Concentração) que devem ser avaliados em uma escala de sete pontos, tipo Likert, variando de 1 (nunca) a 7 (sempre), e ponto médio 4 (algumas vezes). A dimensão vigor é composta por seis itens (e.g., Sinto-me energizado no meu trabalho.), a dedicação por cinco itens (e.g., $\mathrm{Eu}$ considero meu trabalho repleto de significado e propósito.) e a absorção por seis itens (e.g., Não vejo o tempo passar quando estou trabalhando.). $\mathrm{O}$ índice de consistência interna para o fator geral é de $0,95 \mathrm{e}$ para os subfatores variam de 0,85 a 0,87 (Vazquez et al., 2015).

Escala Fatorial de Ajustamento Emocional Neuroticismo - EFN (Hutz \& Nunes, 2001): utilizada para avaliar os índices de neuroticismo e suas dimensões vulnerabilidade, desajustamento psicossocial, ansiedade e depressão. $\mathrm{O}$ instrumento é composto por 82 itens que devem ser avaliados em uma escala Likert de sete pontos, variando de 1 (se a pessoa discorda plenamente que a afirmação descreve uma característica sua) a 7 (se o participante concorda totalmente que a afirmação o descreve de forma adequada). A dimensão vulnerabilidade é composta por 23 itens, desajustamento psicossocial por 25 itens e depressão por 20 itens. Os índices de consistência interna variam de 0,82 a 0,93 (Hutz \& Nunes, 2001).

Self-Reporting Questionnaire - SRQ-20 (Santos, Araújo \& Oliveira, 2009): este instrumento é utilizado para avaliar os transtornos mentais comuns, podendo 
ser utilizado no âmbito ocupacional. O questionário multidimensional é composto por 20 itens que avaliam a existência ou inexistência de quatro grupos de sintomas: humor depressivo-ansioso, sintomas somáticos, decréscimo de energia vital e pensamentos depressivos. A dimensão humor depressivo-ansioso é composta por seis itens (e.g., Sente-se triste ultimamente?), para avaliar os sintomas somáticos são utilizados quatro itens (e.g., Você sente desconforto estomacal), a dimensão decréscimo de energia vital é formada por quatro itens (e.g., O seu trabalho traz sofrimento?) e pensamentos depressivos são avaliados por seis itens (e.g., Tem dificuldade de tomar decisão?). O coeficiente de consistência interna geral é de 0,80 (Santos et al., 2009).

\section{Procedimento de coleta de dados}

Este estudo faz parte de um projeto maior intitulado "Estratégias positivas em Saúde do Trabalhador: um estudo sobre recursos pessoais, engajamento e Burnout em trabalhadores do Judiciário no Rio Grande do Sul"; aprovado por Comitê de Ética (Parecer 511.720).

Em colaboração com o Tribunal de Justiça do Estado do Rio Grande do Sul e com o Centro de Apoio e Desenvolvimento Humano da Comarca da Capital, os servidores foram contatados individualmente através do e-mail institucional. A coleta foi realizada por meio de uma plataforma online, sendo que os participantes responderam voluntariamente aos instrumentos em seu próprio local de trabalho. Os participantes obtiveram acesso aos instrumentos apenas após lerem e concordarem com o Termo de Consentimento Livre e Esclarecido (TCLE), apresentado no formato online apresentado a todos os participantes. No TCLE foi assegurado aos participantes o sigilo dos dados, que sua participação era voluntária e que eles poderiam parar de colaborar com a pesquisa em qualquer momento sem qualquer dano ou prejuízo para eles. A coleta ocorreu no período julho a dezembro de 2014.

\section{Procedimentos para análise dos dados}

O tratamento quantitativo dos dados foi realizado por meio do software SPSS, versão 21. Após a obtenção dos escores totais das dimensões foram verificados os pressupostos de normalidade, homocedasticidade e esfericidade. Os três pressupostos foram atendidos nas dimensões avaliadas (Kolmogorov-Smirnov, $p>0,01$; Shapiro-Wilk, $p>0,01$; KMO $>0,800$, Teste de esfericidade de Bartlett, $p<0,001)$. Os casos com dados faltantes (missings) foram excluídos das análises.

Foram realizadas análises de correlações bivariadas para investigar as relações do engajamento no trabalho e suas dimensões (vitalidade, dedicação e concentração), da saúde mental e suas das dimensões (humor depressivo-ansioso, sintomas somáticos, decréscimo de energia vital e pensamentos depressivos) e das dimensões do neuroticismo (vulnerabilidade, desajustamento psicossocial, ansiedade e depressão). Foi investigado se as dimensões do neuroticismo, os escores de saúde mental e suas dimensões poderiam predizer os índices de engajamento. As análises de regressão citadas foram realizadas por meio da análise de regressão hierárquica (método stepwise). O tamanho da amostra foi satisfatório para o desenvolvimento das regressões citadas (Miles \& Shevlin, 2001), pois era esperado que as relações entre os preditores (dimensões do neuroticismo, os escores de saúde mental e suas dimensões) e o desfecho (engajamento) apresentassem um tamanho de efeito médio.

Posteriormente, foram conduzidas análises de rede (Lauritzen, 1996) por meio do pacote qgraph (Epskamp, Cramer, Waldorp, Schmittmann, \& Borsboom, 2012) do software estatístico $R$. A análise de rede é um método exploratório com base na teoria dos grafos. Nesta técnica uma matriz de adjacência é representada por meio de um objeto gráfico. Nesse gráfico as variáveis são representadas por vértices (ou círculos) e as relações entre as variáveis como arestas (ou linhas). A intensidade das arestas do grafo representa a magnitude dessas associações enquanto sua cor, vermelha ou verde, representam a direção (negativa ou positiva, respectivamente) das associações. O grafo ainda conta com a aplicação de um algoritmo de posicionamento (Fruchterman \& Reingold, 1991), no qual as variáveis são aproximadas ou expelidas de acordo com sua associação. As variáveis representadas no centro do grafo possuem maior número de associações (Machado, Vicossi \& Epskamp, 2015).

Neste estudo, foi gerado um grafo indicando as correlações parciais (ou Campo Aleatório de Markov; Lauritzen, 1996), isto é, associações par a par após o controle estatístico das demais variáveis do modelo (i.e. condicionais). Para evitar o super-ajuste do modelo aos dados é utilizado um hiperparâmetro de penalização por meio do método Graphical Least Abssolute Shrinkage and Selection Operator (GeLasso) (Friedman, Hastie \& Tibshirani, 2008) que fixa em zero arestas com magnitudes próximas a zero. A escolha do melhor modelo se dá por meio do índice de ajuste Extended Bayesian Criterion (EBIC) para gerar o grafo com menor resíduo (Foygel \& Drton, 2010). Por fim, foram estimados os caminhos mais curtos de associação (shortest path) entre as variáveis investigadas a fim de determinar se possuem relações diretas ou mediadas no modelo (Opsahl, Agneessens, \& Skvoretz, 2010). 


\section{Resultados}

Com relação ao escore geral do engajamento no trabalho, os oficiais de justiça apresentaram média de 4,9 (percentil 55; $D P=1,1$ ), o que indica que estão acima do ponto médio da escala, caracterizando-se como profissionais engajados em seu trabalho. Do mesmo modo, as dimensões vigor $(M=4,9$; percentil 60; $D P=1,1)$, dedicação $(M=4,9$; percentil $50 ; D P=1,5)$ e concentração $(M=4,8$; percentil $60 ; D P=1,0)$ obtiveram resultados medianos.

Dentre os índices da EFN, verificou-se que a Depressão foi a dimensão que atingiu o maior índice médio, sendo 47,0 (percentil $70 ; D P=24,0$ ) para o sexo feminino e 42,9 (percentil $60 ; D P=22,2$ ) para o masculino. Resultados aos itens específicos desta dimensão indicaram que 10 participantes apresentavam ideação suicida e 32 desesperança. Verificaram-se baixos índices nas demais dimensões: vulnerabilidade (percentil 35 para mulheres e 25 para homens), desajustamento psicossocial (percentil 35 para mulheres e 45 para homens) e ansiedade (percentil 35 para mulheres e 25 para homens).

A saúde geral, avaliada pelo $S R Q-20$, identificou que $40,1 \%$ dos oficiais de justiça relataram ter algum problema de saúde $(n=33)$. Dentre estes profissionais, cinco $(6,1 \%)$ apresentaram diminuição de energia vital, que trata especificamente de aspectos relativos ao sofrimento no trabalho, dificuldade em manter satisfação com as tarefas realizadas, cansaço, sentimento de inutilidade, dificuldade em pensar com clareza e em tomar decisões. Ademais, 62,2\% dos respondentes referiram sentirem-se tensos e preocupados, $50 \%$ informaram que dormem mal e se cansam com facilidade, $45,1 \%$ disseram que encontram dificuldades para realizar com satisfação suas atividades, e 41,5\% relataram que têm se sentindo triste ultimamente, o que corrobora os resultados obtidos pela dimensão Depressão da EFN. Estes dados são um alerta para o Judiciário visando o bem-estar dessa categoria ocupacional.

\section{Análises de correlação}

As correlações bivariadas apontaram relações significativas negativas entre o engajamento no trabalho e as dimensões da EFN, bem como a saúde mental (e suas dimensões), sendo a dimensão de pensamentos depressivos a que apresentou o coeficiente de maior magnitude. As correlações também apontaram relações positivas e significativas entre saúde mental (e suas dimensões) e as dimensões da EFN (Tabela 1).

\section{Análises de Regressão}

Para analisar o poder de predição das variáveis independentes sobre o engajamento no trabalho foi realizada análise de regressão hierárquica. No primeiro bloco da regressão foram incluídas as dimensões do neuroticismo (vulnerabilidade, desajustamento psicossocial, ansiedade e depressão). No segundo bloco, optou-se pela variável de saúde mental e suas dimensões, uma vez que as correlações revelaram-se mais altas para estas variáveis (critério estatístico). A Tabela 2 mostra o resultado obtido nas regressões.

O modelo final (Modelo 2) da análise apresentou índices de ajuste adequados $(\mathrm{F}(3,197)=195,17 p \leq 0,001)$, explicando $27 \%$ dos índices de engajamento. Os resultados demonstraram que a dimensão decréscimo de energia vital foi identificada como a melhor preditora

\section{TABELA 1}

Correlações Bivariadas entre as Dimensões de Saúde Mental (Humor Depressivo-Ansioso, Sintomas Somáticos, Decréscimo de Energia Vital e Pensamentos Depressivos), Neuroticisimo (Vulnerabilidade, Desajustamento Psicossocial, Ansiedade e Depressão) e Engajamento no Trabalho

\begin{tabular}{|c|c|c|c|c|c|c|c|c|}
\hline Dimensões & 1 & 2 & 3 & 4 & 5 & 6 & 7 & 8 \\
\hline \multicolumn{9}{|l|}{ 1. SRQ_HDA } \\
\hline 2. SRQ_SS & $0,69^{* *}$ & & & & & & & \\
\hline 3. SRQ_DEV & $0,62 * *$ & $0,62 * *$ & & & & & & \\
\hline 4. SRQ_PD & $0,69^{* *}$ & $0,70^{* *}$ & $0,77^{* *}$ & & & & & \\
\hline 5. EFN_N1 & $0,56^{* *}$ & $0,39 * *$ & $0,56^{* *}$ & $0,49^{* *}$ & & & & \\
\hline 6. ENF_N2 & $0,23^{*}$ & $0,26^{*}$ & $0,23^{*}$ & $0,31^{* *}$ & $0,57^{* *}$ & & & \\
\hline 7. EFN_N3 & $0,61 * *$ & $0,53 * *$ & $0,57 * *$ & $0,59 * *$ & $0,79 * *$ & $0,64 * *$ & & \\
\hline 8. EFN_N4 & $0,51^{* *}$ & $0,33^{* *}$ & $0,59^{* *}$ & $0,58^{* *}$ & $0,76^{* *}$ & $0,61^{* *}$ & $0,74^{* *}$ & \\
\hline 9. ENG_T & $-0,43 * *$ & $-0,23 *$ & $-0,51 * *$ & $-0,53 * *$ & $-0,23 *$ & $-0,05$ & $-0,32 * *$ & $-0,38^{* *}$ \\
\hline
\end{tabular}

$* p \leq 0,05 ; * * p \leq 0,001$.

SRQ_HDA=humor depressivo-ansioso; SRQ_SS=sintomas somáticos; SRQ_DEV=decréscimo de energia vital; SRQ_PD=pensamentos depressivos;

ENF_N1=vulnerabilidade; ENF_N2=desajustamento psicossocial; ENF_N3=ansiedade; ENF_N4=depressão; ENG_T=engajamento no trabalho. 
do engajamento no trabalho $\left(R^{2}=0,27 ; \beta=-0,40\right.$; $p \leq 0,05)$. Ainda que menor, a dimensão desajustamento psicossocial também ofereceu contribuição, bem como a depressão. As demais dimensões não contribuíram para explicar este modelo.

Destaca-se que as relações da dimensão desajustamento psicossocial com os índices de engajamento observadas na regressão hierárquica contradizem a associação destas variáveis nas correlações bivariadas. Estas diferenças de resultados envolvendo as mesmas variáveis em análises similares podem resultar do efeito de supressão (Paulhus, et al., 2004). O efeito de supressão ocorre quando o efeito indireto é tão forte que, em uma análise de correlação, ele supera o efeito direto. Desta forma, o efeito de supressão foi observado, mostrando que na análise correlacional o efeito indireto do desajustamento psicossocial com a depressão, decréscimo de energia vital e engajamento dificulta o efeito direto do desajustamento psicossocial sobre os indicadores de engajamento. É importante destacar que o efeito direto é mais informativo que o efeito indireto, pois considera a relação entre as variáveis considerando os diferentes fatores que impactam nessa relação (Paulhus et al., 2004).

\section{Análises de Rede}

Na Figura 1 está representada a rede de correlações parciais, ou seja, das relações par a par após controlados os efeitos das demais variáveis investigadas. Em comparação com as correlações bivariadas, percebe-se que esta rede mantém apenas aquelas relações menos dependentes e mais estáveis neste sistema. É possível destacar que as variáveis decréscimo de energia vital e pensamentos depressivos possuem maior associação com o engajamento no trabalho. Ainda, as variáveis desajustamento psicossocial e depressão possuem relações diretas e de pequena magnitude com engajamento. A depressão possui ainda uma associação maior com as dimensões de saúde decréscimo de energia vital e pensamentos depressivos.

\section{TABELA 2}

Regressão Múltipla Hierárquica das Dimensões de Neuroticisimo (Vulnerabilidade, Desajustamento Psicossocial, Ansiedade e Depressão) e Saúde Mental (Humor Depressivo-Ansioso, Sintomas Somáticos, Decréscimo de Energia Vital e Pensamentos Depressivos) como Preditores do Engajamento no Trabalho como Desfecho

\begin{tabular}{llcccc}
\hline \multicolumn{1}{c}{ Modelo/Passo } & & $R^{2}$ & $R^{2} A d j$. & $\Delta R^{2}$ Adj. & $\beta$ \\
\hline Modelo 1/Passo 1 & Vulnerabilidade & 0,19 & 0,17 & 0,05 & - \\
& $\begin{array}{l}\text { DesajustamentoPsicossocial } \\
\text { Ansiedade }\end{array}$ & & & $-0,20^{*}$ \\
& Depressão & & & - \\
Modelo 1/Passo 2 & Humor Depressivo-Ansioso & 0,29 & 0,27 & 0,10 & $-0,27^{*}$ \\
& Sintomas Somáticos & & & - \\
& Decréscimo de Energia Vital & & & $-0,40^{*}$ \\
Modelo 2/Passo 1 & Pensamentos Depressivos & & & - \\
Modelo 2/Passo 2 & DesajustamentoPsicossocial & 0,19 & 0,17 & 0,05 & $-0,20^{*}$ \\
Modelo 2/Passo 3 & Decréscimo de Energia Vital & 0,14 & 0,13 & 0,14 & $-0,27^{*}$ \\
\hline
\end{tabular}

$* p \leq 0,05$

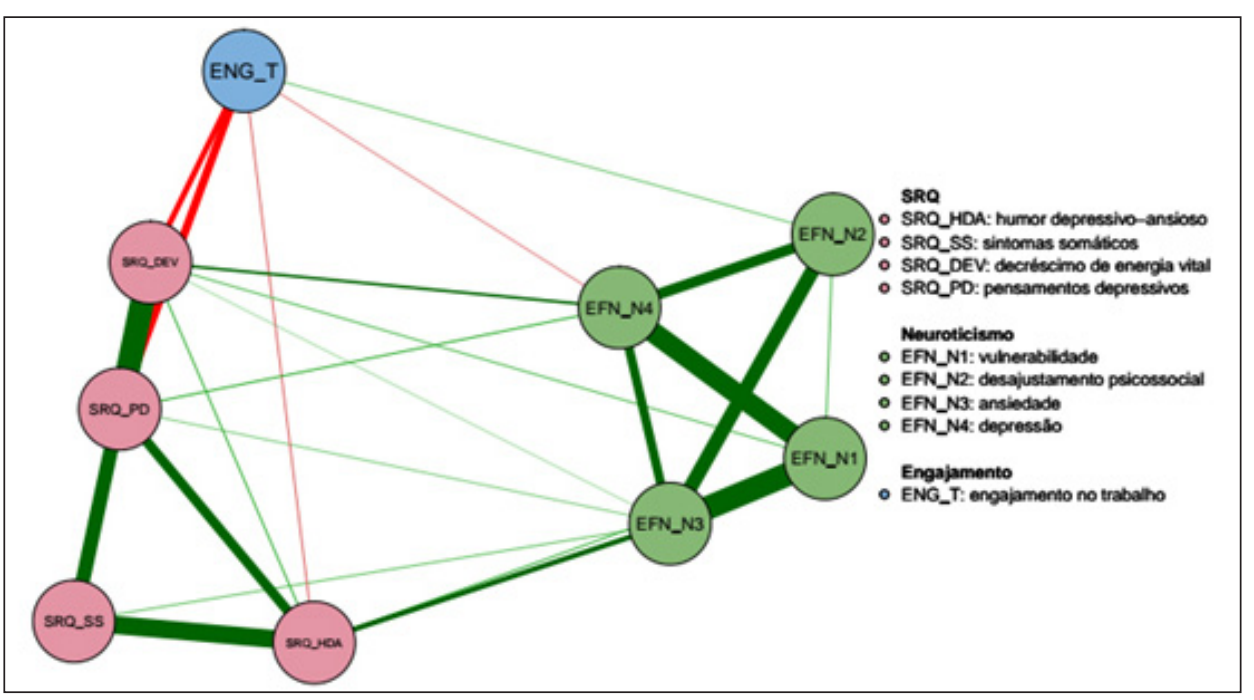

Figura 1. Análise de Rede Método Glasso. 
TABELA 3

Matriz de Predição e Caminhos Mínimos entre as Dimensões de Saúde Mental (Humor Depressivo-Ansioso, Sintomas Somáticos, Decréscimo de Energia Vital e Pensamentos Depressivos), Neuroticisimo (Vulnerabilidade, Desajustamento Psicossocial, Ansiedade e Depressão) e Engajamento no Trabalho

\begin{tabular}{lccccccccc}
\hline \multicolumn{1}{c}{ Dimensões } & 1 & 2 & 3 & 4 & 5 & 6 & 7 & 8 & 9 \\
\hline 1. SRQ_HDA & & 0,3 & 0,08 & 0,2 & 0,08 & & 0,15 & & $-0,06$ \\
2. SRQ_SS & 2,1 & & 0,11 & 0,26 & & & 0,06 & & \\
3. SRQ_DEV & $3,4,1$ & $3,4,2$ & & 0,38 & 0,07 & & 0,03 & 0,11 & $-0,15$ \\
4. SRQ_PD & 4,1 & 4,2 & 4,3 & & & & 0,05 & 0,09 & $-0,18$ \\
5. EFN_N1 & $5,7,1$ & $5,7,1,2$ & $5,8,3$ & $5,8,3,4$ & & 0,07 & 0,37 & 0,34 & \\
6. EFN_N2 & $6,7,1$ & $6,7,1,2$ & $6,8,3$ & $6,7,1,4$ & $6,7,5$ & & 0,26 & 0,21 & 0,06 \\
7. EFN_N3 & 7,1 & $7,1,2$ & $7,8,3$ & $7,1,4$ & 7,5 & 7,6 & & 0,19 & \\
8. EFN_N4 & $8,7,1$ & $8,3,4,2$ & 8,3 & $8,3,4$ & 8,5 & 8,6 & 8,7 & & $-0,05$ \\
9. ENG_T & $9,4,1$ & $9,4,2$ & 9,3 & 9,4 & $9,3,8,5$ & 9,6 & $9,4,1,7$ & $9,3,8$ & \\
\hline
\end{tabular}

SRQ_HDA=humor depressivo-ansioso; SRQ_SS=sintomas somáticos; SRQ_DEV=decréscimo de energia vital; SRQ_PD=pensamentos depressivos; $\mathrm{N} 1=$ vulnerabilidade; N2=desajustamento psicossocial; N3=ansiedade; N4=depressão; ENG_T=engajamento no trabalho.

Valores faltantes na diagonal superior foram fixados em zero pelo método de regularização.

Por fim, a Tabela 3 indica o caminho mais curto de associação (shortest path) entre as variáveis investigadas. O modelo gráfico indica que as variáveis decréscimo de energia vital, pensamentos depressivos e desajustamento psicossocial possuem uma ligação direta com o engajamento, enquanto as variáveis humor depressivo-ansioso, sintomas somáticos, vulnerabilidade, ansiedade e depressão possuem relações mediadas, ou seja, indiretas com o desfecho investigado.

\section{Discussão}

O principal objetivo deste estudo foi avaliar os níveis de engajamento no trabalho, do traço de personalidade neuroticismo e da saúde mental de servidores do Judiciário e investigar as relações entre estes construtos. As análises descritivas indicaram que o engajamento dos oficiais de justiça do TJRS é mediano. Foi observado que apesar da grande carga emocional, falta de reconhecimento e das demais dificuldades relacionadas a esta profissão (Merlo et al., 2012; Pereira \& Assunção, 2007; Pizzinato et al., 2014), os oficiais de justiça parecem despender esforços de modo persistente (vigor) para obter desempenho além do esperado (dedicação) e sentem-se absorvidos em seu trabalho de maneira prazerosa e desafiadora (concentração). Este resultado pode ser explicado pela presença de determinados recursos do trabalho (e.g., reconhecimento social, autonomia, apoio social) que levam a uma dedicação maior na realização de suas tarefas, mesmo com as altas demandas enfrentadas diariamente pelos oficiais de justiça.

Por ser uma profissão em grande parte solitária, não há o reconhecimento das atividades dos oficiais de justiça por parte dos juízes e nem mesmo pelos colegas (Pizzinato et al., 2014; Merlo, Dornelles, Bottega, \& Trentini, 2012; Tavares, 2003). No entanto, o estudo realizado por Pereira e Assunção (2007) com servidores do Judiciário demonstrou o orgulho destes profissionais pelo reconhecimento externo que obtêm dos cidadãos, por serem vistos como "porta-vozes da justiça”, com importância social. Tal reconhecimento é expresso pelo bom relacionamento, confiança e receptividade com o público, que os auxilia até mesmo nas buscas por pessoas e endereços. Ainda que possa ser associado à periculosidade, o contato com o público é descrito como motivador (Pizzinato et al., 2014). Assim, este reconhecimento acaba desempenhando um papel de feedback para os oficiais, citado na literatura como um recurso de trabalho adequado (Hu, Schaufeli, \& Taris, 2011; Schaufeli \& Taris, 2014).

No mesmo estudo, os oficiais relataram satisfação por terem autonomia quanto a organização do trabalho e do tempo na realização de suas atividades diárias (Pereira \& Assunção, 2007). A autonomia tem sido citada por diversos autores como um importante recurso do trabalho no aumento dos níveis de engajamento de profissionais (Hu et al., 2011; Schaufeli \& Taris, 2014). Ademais, outro fator que poderia explicar este resultado é a satisfação pelo salário $(94,3 \%)$, estabilidade $(87,3 \%)$ e benefícios recebidos $(42,2 \%)$ evidenciadas no estudo de Merlo et al. (2012). Portanto, apesar da alta demanda de trabalho caracterizada pela pressão exercida pelo volume de mandatos e pelos riscos físico e mental/emocional das situações enfrentadas, os oficiais dispõem de alguns recursos que ainda os mantêm engajados. 
Em consonância com pesquisas anteriores, este estudo demonstrou associação significativa entre saúde e engajamento no trabalho (Hakanen \& Schaufeli, 2012; Innstrand, Langballe, \& Falkum, 2012). Todas as dimensões do questionário de saúde obtiveram correlação negativa com as dimensões do engajamento, ou seja, oficiais de justiça que revelam sintomas psiquiátricos menores tendem a apresentar menores níveis de engajamento. Um estudo longitudinal (Hakanen \& Schaufeli, 2012) verificou que o engajamento pode predizer negativamente sintomas de depressão, concluindo que o engajamento pode ser um fator de proteção contra problemas psicológicos. De modo similar, a dimensão vigor do engajamento foi descrita como preditora de níveis menores de sintomas de depressão e ansiedade no estudo de Innstrand, Langballe e Falkun (2012) que caracterizou o engajamento como um possível modificador da saúde em diferentes grupos ocupacionais noruegueses.

Mesmo o engajamento sendo apontado como um fator de saúde e bem-estar (Hakanen \& Schaufeli, 2012; Innstrand et al., 2012), os resultados do questionário de saúde geral identificaram um grande número de oficias de justiça com problemas de saúde. $\mathrm{Na}$ literatura, os escassos estudos com servidores do judiciário também revelam resultados semelhantes. Fatores de saúde ocupacional (sintomas físicos e psicológicos), como dores de cabeça, dores nas costas e depressão, foram relatados pelos trabalhadores do judiciário dos grupos focais realizados por Pizzinato et al. (2014). Ao investigar os efeitos do trabalho sobre a saúde de oficiais de justiça, Merlo et al. (2012) identificou que 50,7\% desta população apresentou distúrbios psiquiátricos menores, sendo que $7,1 \%$ dos respondentes relataram ideação suicida e $8,6 \%$ referiram se sentir incapazes de desempenhar um papel útil em sua vida. Estes resultados também se refletiram no percentual relevante de servidores que utilizavam antidepressivos $(36,1 \%)$, ansiolíticos $(19,1 \%)$ ou medicamentos para dormir $(19,1 \%)$.

$\mathrm{Na}$ mesma direção, dentre as dimensões da escala de neuroticismo, a depressão apresentou altos índices em nossos participantes, caracterizando-os como profissionais com baixa expectativa em relação ao seu futuro que relatam ter uma vida monótona e sem objetivos claros, e que se consideram pessoas solitárias. Ressalta-se a importância destes dados, que corroboram com os demais estudos sobre a saúde dos servidores do judiciário, visto que apontam casos de depressão grave e de ideação suicida, sendo necessário intervenção imediata. Uma vez que estes oficiais de justiça apresentam sérios problemas de saúde, é possível inferir que o custo para estes profissionais se manterem engajados em um contexto com tantas situações adversas esteja repercutindo em sua saúde física e mental.

Em relação às demais dimensões da escala de neuroticismo, os baixos escores de vulnerabilidade e desajustamento psicossocial demonstram que os oficiais de justiça são bem menos vulneráveis e menos sensíveis às situações alheias do que a maior parte da população. Estes resultados podem estar atribuídos ao fato de que para ser oficial de justiça talvez seja necessário possuir características como frieza, falta de sensibilidade e distanciamento dos relacionamentos sociais para conseguir lidar com o contexto de risco diário em que são expostos. Tavares (2003) evidenciou comportamento similar em servidores do judiciário que demonstraram distanciamento afetivo do trabalho, citado como estratégia para amenizar o impacto do grande volume de processos que se acumulavam, dos quais não conseguiam dar conta. A responsabilidade sentida por eles ainda era acentuada por lidarem com processos que se referiam ao interesse de pessoas em situação de carência. Assim, o distanciamento afetivo foi a estratégia encontrada para não sofrerem com esta rotina.

Ao contrário do que se esperava, tendo em vista o tipo de trabalho realizado pelos oficiais de justiça, foram encontrados escores de ansiedade muito baixos. O que significa que eles se sentem pouco ansiosos diante de situações adversas e riscos reais, possivelmente como estratégia psicológica para se manter na função. Os escores muito baixos em ansiedade indicam falta de adaptação, pois envolvem situações que possam oferecer algum risco (físico ou psicológico), sem se colocarem suficientemente em alertas para todos os novos elementos com os quais tem que lidar (Hutz \& Nunes, 2001). Importante ressaltar que este tipo de comportamento não caracteriza 'desatenção' com as atividades, mas falta de motivação e, possivelmente, naturalização dos riscos associados ao cotidiano de trabalho em atividades externas, como situações de violência urbana e acidentes de trajeto. As dimensões do neuroticismo tiveram correlação negativa e significativa com o engajamento, como observado em outras amostras (Lin et al., 2016; Sulea et al., 2015; Zis et al., 2016). O alto nível de neuroticismo está associado a indivíduos que tendem a avaliar de forma negativa seu ambiente e a experienciar mais intensamente sofrimento emocional (Hutz \& Nunes, 2001), comprometendo a maneira como percebe seu contexto laboral e, consequentemente, o engajamento em seu trabalho.

Em relação à identificação dos preditores do engajamento, os resultados desta pesquisa demons- 
traram que o decréscimo de energia vital foi o melhor preditor desta variável. À medida que diminui o índice de decréscimo de energia vital dos servidores, ou seja, que diminui o sofrimento, a insatisfação, o sentimento de inutilidade e o cansaço no trabalho, aumenta-se a possibilidade dos oficiais de justiça estarem engajados. Tais constatações reforçam a ideia de que o engajamento no trabalho possui uma importante relação com bem-estar e saúde mental (Hakanen \& Schaufeli, 2012; Innstrand et al., 2012). Apesar do modelo estudado nesta pesquisa ter apresentado os fatores de saúde como preditores do engajamento, há na literatura científica o entendimento de que estes podem ser consequência do engajamento. (Hakanen \& Schaufeli, 2012; Innstrand et al., 2012).

As dimensões do traço de personalidade neuroticismo relativas à depressão e ao desajustamento psicossocial demonstraram ser preditoras menos importantes do engajamento, do que os fatores de saúde. Diferente do esperado, o desajustamento psicossocial foi evidenciado na análise de regressão influenciando positivamente os índices de engajamento nos participantes da pesquisa. O desajustamento psicossocial é caracterizado por indivíduos que tendem a ser agressivos com os outros, que relatam gostar de envolver-se em situações de risco, frequentemente manipulando situações de tal forma que os beneficie (Hutz \& Nunes, 2001). Tratando-se de uma amostra específica de oficias de justiça, que lida com condições de trabalho inadequadas e cujas funções exigem ações sociais mandatórias, este resultado pode ser explicado pelo processo de adaptação dos profissionais à função. $\mathrm{Na}$ rotina dos oficiais de justiça isso se expressa por visitarem sozinhos locais perigosos onde precisam entregar os mandados, como em comunidades com altos índices de vulnerabilidade social, além de enfrentarem reações de agressões verbais e físicas (Pereira \& Assunção, 2007).

Com relação às análises de rede, controlados o efeito das demais variáveis, a dimensão depressão demonstrou uma relação indireta com o engajamento, que foi mediada pela saúde mental. Os resultados mostram que as facetas de personalidade que entraram no modelo de regressão e na análise de redes apresentam relação indireta na explicação do engajamento.

De modo geral, as dimensões da personalidade apresentaram associação fraca com o engajamento. De modo similar, Maas e Spinath (2012), pesquisando a relação entre personalidade e recursos para lidar com as demandas profissionais, demonstraram que as influências genéticas e ambientais destes recursos foram em grande parte independentes dos efeitos genéticos e ambientais na personalidade. Ainda, os resultados deste estudo sugeriram que as experiências durante o desenvolvimento, bem como outras influências ambientais específicas que atuam na personalidade do indivíduo diferem das experiências no local de trabalho que influenciam os comportamentos organizacionais. Desta forma, pressupõe-se que intervenções com oficias de justiça talvez devessem concentrar-se em nível organizacional, na melhoria dos recursos e condições do trabalho.

\section{Considerações finais}

Foi possível verificar o impacto das variáveis de saúde mental e do neuroticismo sobre o engajamento no trabalho. O decréscimo de energia vital foi o melhor preditor em todas as análises. Os resultados mostram a importância dos aspectos relativos ao sofrimento, cansaço e insatisfação no trabalho dos oficias de justiça, impactando negativamente no engajamento.

As análises de rede evidenciaram que a relação da depressão com engajamento é mediada pelas variáveis de saúde, não havendo associação direta entre estes construtos. Ademais, dado que o engajamento é uma característica saudável, não era esperada associação positiva com desajustamento psicossocial no modelo. Sendo assim, é possível que oficiais de justiça que possuam tais características de personalidade estejam mais ajustados a esta função. Sugere-se que esta interpretação seja testada em estudos futuros, inclusive para entender se os indivíduos mais engajados são os mesmos que apresentam tais sintomas.

Considerando os resultados obtidos, o presente estudo evidenciou importantes contribuições referentes à literatura do engajamento no trabalho, demonstrando o papel da saúde mental e das diferenças individuais, em particular as dimensões do neuroticismo, no engajamento de servidores do judiciário. Tais constatações sugerem que intervenções para melhoria dos níveis de engajamento devem considerar programas de promoção de saúde mental no âmbito organizacional, tendo em vista as dificuldades de impactar sobre os fatores individuais, como traços de personalidade.

Apesar de este estudo contribuir para o avanço no entendimento das relações das características da personalidade e saúde mental com o engajamento, isto não ocorre sem limitações. As limitações desta pesquisa foram a impossibilidade de generalização dos resultados devido ao tamanho e especificidade da amostra. Observa-se a necessidade de serem realizados estudos investigando as relações propostas nesta pesquisa com oficiais de justiça de outras regiões do Brasil, assim como outras categoriais profissionais. 


\section{Referências}

Epskamp, S., Cramer, A. O. J., Waldorp, L. J., Schmittmann, V. D., \& Borsboom, D. (2012). Qgraph: Network visualizations of relationships in psychometric data. Journal of Statistical Software, 48, 1-18. https://doi.org/10.18637/ jss.v048.i04

Foygel, R. and Drton, M. (2010). Extended Bayesian information criteria for Gaussian graphical models. Advances in Neural Information Processing Systems, 23, 2020-2028.

Friedman, J., Hastie, T., \& Tibshirani, R. (2008). Sparse inverse covariance estimation with the graphical lasso. Biostatistics, 9(3), 432-441. https://doi.org/10.1093/biostatistics/kxm045

Fruchterman, T. M. J. \& Reingold, E. M. (1991). Graph drawing by force-directed placement. Software - Practice and Experience, 12, 1129-1164. https://doi.org/10.1002/spe.4380211102

Lauritzen, S. L. (1996). Graphical models. Oxford, UK: Clarendon Press,

Hakanen, J. J. \& Schaufeli, W. B. (2012). Do burnout and work engagement predict depressive symptoms and life satisfaction? A three-wave seven-year prospective study. Journal of Affective Disorders, 141, 415-424. https://doi. org/10.1016/j.jad.2012.02.043

Hu, Q., Schaufeli, W. B., \& Taris, T. W. (2011). The Job Demands-Resources model: An analysis of additive and joint effects of demands and resources. Journal of Vocational Behavior, 79(1), 181-190. https://doi.org/10.1016/j. jvb.2010.12.009

Hutz, C. S. \& Nunes, C. H. S. S. (2001). Escala Fatorial de Ajustamento Emocional/Neuroticismo. São Paulo: Casa do Psicólogo. https://doi.org/10.1590/S0102-79722013000200009

Innstrand, S. T., Langballe, E. M., \& Falkum, E. (2012). A Longitudinal Study of the Relationship between Work Engagement and Symptoms of Anxiety and Depression. Stress and Health, 28, 1-10. https://doi.org/10.1002/smi.1395

Lin, W., Wang, L., Bamberger, P. A., Zhang, Q., Wang, H., Guo, W., \& Zhang, T. (2016). Leading future orientations for current effectiveness: The role of engagement and supervisor coaching in linking future work self salience to job performance. Journal of Vocational Behavior, 92, 145-156.

Machado, W. L., Vissoci, J., \& Epskamp, S. (2015). Análise de rede aplicada à Psicometria e à Avaliação Psicológica. In C. S. Hutz, D. R. Bandeira, \& C. M. Trentini (Orgs.). Psicometria (pp. 125-146). Porto Alegre: ArtMed.

Maas, H. \& Spinath, F. M. (2012). Personality and Coping with Professional Demands: A Behavioral Genetics Analysis. Journal of Occupational Health Psychology, 17(3), 376-385. https://doi.org/10.1037/a0027641

Merlo, A. R. C., Dornelles, R. A. N., Bottega, C. G., \& Trentini, L. (2012). O trabalho e a saúde dos oficiais de Justiça Federal de Porto Alegre. Cadernos de Psicologia Social do Trabalho, 15(1), 101-113. https://doi.org/10.11606/ issn.1981-0490.v15i1p101-113

Miles, J. \& Shevlin, M. (2001). Applying Regression and Correlation: A Guide for Students and Researchers, Thousand Oaks: Sage.

Ministério da Previdência Social. (2015). Acompanhamento mensal dos benefícios auxílios-doença previdenciários concedidos segundo os códigos da CID-10. Recuperado de: http://www.mtps.gov.br/arquivos/office/4_100111115547-873.pdf

Opsahl, T., Agneessens, F., \& Skvoretz, J. (2010). Node centrality in weighted networks: Generalizing degree and shortest paths. Social Networks, 23, 245-251. https://doi.org/10.1016/j.socnet.2010.03.006

Paulhus, D. L., Robins, R. W., Trzesniewski, K. H., \& Tracy, J. L. (2004). Two replicable suppressor situations in personality research. Multivariate Behavioral Research, 39(2), 303-328. https://doi.org/10.1207/s15327906mbr3902_7

Pereira, P. V. A. \& Assunção, A. A. (2007). Trabalho e emoção: o caso dos oficiais de justiça. Pesquisas e Práticas Psicossociais, 2(1). Recuperado de: http://www.ufsj.edu.br/portal2-repositorio/File/revistalapip/11artigo.pdf

Pizzinato, A., Carlotto, M. S., Cé, J. P., \& Conceição, I. K. (2014). Significados do trabalho e qualidade de vida percebida em trabalhadores do judiciário. Revista Interinstitucional de Psicologia, 7 (2), 188-198. Recuperado de: http://pepsic. bvsalud.org/pdf/gerais/v7n2/v7n2a07.pdf

Santos, K. O. B., Araújo, T. M., \& Oliveira, N. F. (2009). Estrutura fatorial e consistência interna do Self-Reporting Questionnaire (SRQ-20) em população urbana. Cadernos de Saúde Pública, 25(1), 214-222. https://doi.org/10.1590/ S0102-311X2009000100023

Schaufeli, W. B. (2013). What is engagement? In C. Truss, K. Alfes, R. Delbridge, A. Shantz, \& E. Soane (Eds.). Employee Engagement in Theory and Practice. London: Routledge.

Schaufeli, W. B., Dijkstra, P., \& Vazquez, A. C. (2013). Engajamento no trabalho. São Paulo: Casa do Psicólogo.

Schaufeli, W. B., \& Taris, T.W. (2014). A critical review of the Job Demands-Resources Model: Implications for improving work and health. In G. F. Bauer \& O. Hämmig (Eds.). Bridging occupational, organizational and public health (pp. 43-68). Amsterdam: Springer.

Sulea, C., Van Beek, I., Sarbescua, P., Virga, D., \& Schaufeli, W. B. (2015). Engagement, boredom, and burnout among students: Basic need satisfaction matters more than personality traits. Learning and Individual Differences, 42, 132-138. https://doi.org/10.1016/j.lindif.2015.08.018 
Tavares, D. S. (2003). Sofrimento psíquico entre servidores públicos: uma análise psicossocial do contexto do trabalho em um Tribunal Judiciário Federal. Dissertação de mestrado não-publicada, Universidade de São Paulo, São Paulo, Brasil.

Vazquez, A. C. S., Magnan, E. S., Pacico, J. C., Hutz, C. S., \& Schaufeli, W. B. (2015). Adaptation and Validation of the Brazilian Version of the Utrecht Work Engagement Scale. Psico-USF, 20(2), 207-217. https://doi.org/10.1590/141382712015200202

Zis, P., Anagnostopoulos, F., \& Artemiadis, A. K. (2016). Residency Training: Work engagement during neurology training. Neurology, 87(5), e45-e48. https://doi.org/10.1212/WNL.0000000000002911

\section{Autores:}

Natália dos Santos Dalanhol - Psicóloga, Universidade Federal de Ciências da Saúde de Porto Alegre.

Clarissa Pinto Pizarro de Freitas - Doutora, Universidade Salgado de Oliveira.

Wagner de Lara Machado - Doutor, Pontifícia Universidade Católica de Campinas.

Claudio Simon Hutz - Doutor, Universidade Federal do Rio Grande do Sul.

Ana Claudia Souza Vazquez - Doutora, Universidade Federal de Ciências da Saúde de Porto Alegre.

Endereço para correspondência:

Ana Claudia Souza Vazquez

Rua Sarmento Leite, 245, sala 208

90050-170, Porto Alegre, RS, Brasil

<vazquez.ac@gmail.com>

Recebido em: 27.11.2016

Aceito em: 07.03.2017 